\title{
Risk Factors for In-hospital Progression of Ordinary COVID-19 in Wuhan, China: A Retrospective Cohort Study
}

\section{Ying Zhang}

General Hospital of Central Theatre Command

\section{Xing Fan}

General Hospital of Central Theatre Command

\section{Yan Zhao}

General Hospital of Central Theatre Command

\section{Fan Zhou}

General Hospital of Central Theatre Command

\section{Linlin Zhao}

General Hospital of Central Theatre Command

Jing Liu

General Hospital of Central Theatre Command

Haichao Liu

General Hospital of Central Theatre Command

Chengrui Zhou

General Hospital of Central Theatre Command

\section{Ling Tong}

General Hospital of Central Theatre Command

\section{Zhenhua Lv}

General Hospital of Central Theatre Command

\section{Ying Xiong}

General Hospital of Central Theatre Command

\section{Yong Zhang}

General Hospital of Central Theatre Command

\section{Yingjun Tan}

General Hospital of Central Theatre Command

Airong Yu ( $\nabla$ yarfwy@163.com )

General Hospital of Central Theatre Command 
Keywords: COVID-19, Ordinary, Risk factors, In-hospital progression, Treatment

Posted Date: April 15th, 2020

DOI: https://doi.org/10.21203/rs.3.rs-23094/v1

License: (c) (1) This work is licensed under a Creative Commons Attribution 4.0 International License. Read Full License 


\section{Abstract}

Objective: To describe the clinical characteristics and outcomes of ordinary COVID-19 when admitted, to describe how these patients were treated and risk factors for in-hospital progression.

Methods: In this retrospective study, we included 291 adult patients diagnosed as ordinary COVID-19 on admission who had been discharged or had died between Jan 20, 2020 and Mar 16, 2020 from General Hospital of Central Theatre Command (Wuhan, China).

Results: Of the 291 patients diagnosed as ordinary COVID-19 when admitted, 65 (22.34\%) had been recorded COVID-19 progressing at least once, and 226 (77.66\%) had been recorded COVID-19 improving during hospitalization. The median time from admission to disease progressed was 5.0 days (2.0-7.0). Multivariable regression showed increasing odds of in-hospital progression associated with male (odds ratio 2.333, 95\% $\mathrm{Cl} 1.135-4.395 ; \mathrm{P}=0.020)$, preexisting cardiovascular diseases $(2.433,1.044-5.671$; $P=0.039)$, and lymphopenia (3.482, 1.783-6.799; $P<0.001)$, elevated IL-6 $(2.669,1.084-6.574 ; P=0.033), d-$ dimer $(2.829,1.420-5.636 ; P=0.003)$ and lactate dehydrogenase $(2.855,1.458-5.591 ; P=0.002)$ on admission.

Conclusions: The potential risk factors of male, preexisting cardiovascular disease, lymphopenia, elevated IL-6, and lactate dehydrogenase, d-dimer could help clinicians to identify in-hospital progression among ordinary COVID-19 at early stage to optimize medical treatment.

\section{Introduction}

In December 2019, a group of patients with acute respiratory illness of unknown cause appeared in Wuhan, China, which was confirmed to be caused by a novel corona virus. Full-genome sequencing and phylogenic analysis indicated that the novel corona virus is closely related to bat-derived SARS-like corona viruses ${ }^{1}$ and officially named as severe acute respiratory syndrome coronavirus 2 (SARS-CoV-2) by International Committee on Taxonomy of Viruses. WHO declared the disease caused by SARS-CoV-2 was officially named as corona virus disease 2019 (COVID-19). Epidemiological studies confirmed human-to-human transmission of COVID-192,3. As of Apr 5, 2020, COVID-19 has been described in 208 countries, areas or territories, involving 1,093,349 individuals and causing 58,620 deaths. COVID-19 spread rapidly and has threatened global public health ${ }^{4}$.

The incubation period of COVID-19 infection was 1-14 days, mostly 3-7 days. Most severe patients develop dyspnea and / or hypoxemia one week after onset and may lead to organ dysfunction (such as shock, acute respiratory distress syndrome, septic shock, acute heart injury and acute kidney injury) and death ${ }^{5}$. Huang first reported 41 cases of COVID-19 infection, 22(55\%) developed into dyspnoea (median time from illness onset to dyspnoea 8.0 days (IQR 5-0-13.0), 13 (32\%) patients were admitted to an Intensive Care Unit (ICU) and six (15\%) died ${ }^{6}$. In another single-center case series of 138 COVID19 patients, $26 \%$ received ICU care with a median time of $8.0 \mathrm{~d}$ for initial symptoms to acute respiratory 
distress syndrome (ARDS), and 6 deaths (mortality rate $4.3 \%)^{7}$. Therefore, early detection, early treatment, prevention of disease progression to severity, and reduction of mortality are the focus of clinical diagnosis and treatment.

Current studies have demonstrated that age, sex, comorbidities, some early onset of symptoms and laboratory findings may be risk factors for poor outcome of COVID-19. ${ }^{7-9}$ However, there are some disagreements between those studies. Chen et al found older males with comorbidities were more likely to be affected and resulted in sever and even acute respiratory ARDS ${ }^{8}$, while Wang et al found no male difference between ICU patients and non-ICU patients ${ }^{7}$. A recent study including 78 COVID-19 patients reported several factors led to the progression of COVID-19, but there was no significance in any comorbidities, probably because of the small sample size ${ }^{9}$. Therefore, it is necessary to include more patients to evaluate the possible risk factors associated with COVID-19 progression. As reported that COVID-19 patients were more common to be ordinary cases when admitted to hospital ${ }^{9-11}$, but until now, there is no study focus on ordinary COVID-19 in-hospital progression. So, we represent a quantitative analysis of 291 COVID-19 patients who were clinically classified as ordinary cases on admission to identify possible risk factors for in-hospital progression and hope to provide an insight into the treatment for high-risk severe COVID-19 patients.

\section{Methods}

\section{Ethical approval}

The study was approved by the Research Ethics Commission of General Hospital of Central Theatre Command ([2020]016-1) and the requirement for informed consent was waived by the Ethics Commission.

\section{Study design and participants}

All adult patients included in this retrospective cohort study were diagnosed with COVID-19 laboratoryconfirmed positive for COVID-19 by testing the nucleic acid of respiratory specimens, who were discharged or died between Jan 20, 2020 and Mar 16, 2020. The illness severity of all patients was evaluated and classified clinically upon admission, according to the Chinese management guideline for COVID-19 (version 7.0) ${ }^{5}$. Our study enrolled all patients who were clinically classified as ordinary cases when admitted and had a definite outcome (dead or discharged). We excluded patients whose disease has improved, meanwhile the results for COVID-19 nucleic acids have returned negative before admission, and patients diagnosed as mild, severe or critical on the day of admission.

According to the Chinese management guideline for COVID-19 (version 7.0), the clinical classifications of the disease included: (1) mild cases: the clinical symptoms are mild and no pneumonia manifestation can be found in imaging; (2) ordinary cases: patients have symptoms like fever and respiratory tract symptoms, etc., and pneumonia manifestation can be seen in imaging; (3) sever cases (meeting any of 
the following): respiratory distress, $\mathrm{RR}^{3} 30$ breaths/min; the oxygen saturation is less than $93 \%$ at a rest state; arterial partial pressure of oxygen $\left(\mathrm{PaO}_{2}\right)$ / oxygen concentration $\left(\mathrm{FiO}_{2}\right) £ 300 \mathrm{mmHg}(1$ $\mathrm{mmHg}=0.133 \mathrm{kPa}$ ); (3) critical cases (meeting any of the following): respiratory failure occurs and mechanical ventilation is required monitoring and treatment in ICU ${ }^{5}$.

All patients were evaluated and classified clinically upon admission and during the treatment. Based on the clinical records, patients were included in the disease progression group when the disease progressed to a serious or critical state during the treatment, patients who did not progress were included in the improvement group.

\section{Data collection}

Epidemiological, demographic, clinical, laboratory, radiological, treatment and outcomes data for all patients with confirmed COVID-19 were obtained from electronic medical records by a trained team. We used a standardized case record form to collect clinical data. If information was not clear, the investigators would contact the doctor responsible for the treatment of the patient for clarification.

\section{Laboratory procedures and treatment}

COVID-19 in respiratory specimens was detected by real-time RT-PCR methods. Throat-swab specimens were collected for SARA-CoV-2 re-examination according to the condition of patients (improved or progressive).

Blood count (white blood cell, lymphocyte count), serum biochemical tests (including liver and renal function, myocardial enzymes, lactate dehydrogenase, troponin, myoglobin), C-reactive protein, procalcitonin, interleukin6 (IL6), erythrocyte sedimentation rate and d-dimer were tested with routine blood examinations. Chest imaging was done for all inpatients. The frequency of examinations was based on the patient's condition.

The most common treatment of antivirals included lopinavir/ritonavir, Interferon, Arbidol, and Ribavirin combined with Traditional Chinese Medicine. Patients received glucocorticoid for 3-5 days according to the severity of respiratory distress and the progress of chest imaging. In addition, Respiratory support was used for preventing acute hypoxic respiratory failure.

The criteria for discharge were with normal body temperature for more than 3 days, significantly recovered respiratory symptoms, obvious absorption in both lungs in chest CT, negative results of the nucleic acid tests of respiratory specimens for consecutive two times at least $24 \mathrm{~h}$ apart.

\section{Statistical analysis}

Mean, medians and inter quartile ranges (IQRs) were calculated as continuous variables. Categorical variables were presented as percentages of patients in each category. Independent group t test or MannWhitney $U$ test were used to compare distributions of continuous variables on admission where 
appropriate. The $c^{2}$ test or Fisher exact test were used to compare the proportions for categorical variables where appropriate. Univariate and multivariate logistic regression analysis were adopted to identify risk factors of disease progression. $P<0.05$ (bilateral) was considered statistically significant.

We excluded variables from the univariable analysis if there were no significant difference between the two groups. All statistical analyses were performed using SPSS (Statistical Package for the Social Science) version 22.0 software (SPSS Inc).

\section{Results}

\section{Baseline characteristics}

By Mar 16, 2020, 311 patients were identified as laboratory-confirmed COVID-19, of that 12 (3.8\%) were classified as mild cases, 291(93.6\%) were classified as ordinary cases, and 8(2.6\%) were classified as severe cases when admitted. All have been discharged or died. Finally, the 291 ordinary cases were included in this study(Table 1).The median age of the patients was 56.0 years (IQR 40.0-67.0), ranging from 18 years to 96 years, and most patients were male [163(56.01\%)]. Comorbidities were present in $162(55.67 \%)$ patients, with hypertension being the most common comorbidity, followed by cardiovascular disease and diabetes. The most common symptoms at onset of illness were fever, dry cough, fatigue, chest tightness, and muscle soreness. The median time from onset of symptoms to first hospital admission was 7.0 days (IQR 3.0-11.0).

Table 1: Demographics and clinical characteristics on admission. 


\begin{tabular}{|c|c|c|c|c|}
\hline & Total $(n=291)$ & $\begin{array}{l}\text { progression } \\
\text { group }(n=65)\end{array}$ & $\begin{array}{l}\text { improvement } \\
\text { group }(n=226)\end{array}$ & $\begin{array}{l}P \\
\text { value }\end{array}$ \\
\hline Age, years & $\begin{array}{l}56.0 \otimes 40.0 \\
67.0 \otimes\end{array}$ & $57.0(48.0,71.0)$ & $54.5(38.0,66.0)$ & 0.030 \\
\hline Male & $163(56.01 \%)$ & $47(72.31 \%)$ & $116(51.33 \%)$ & 0.003 \\
\hline $\begin{array}{l}\text { Duration from illness } \\
\text { onset }\end{array}$ & $7(3,11)$ & $6(3,9)$ & $7(3,12)$ & 0.115 \\
\hline \multicolumn{5}{|l|}{ to first admission } \\
\hline \multicolumn{5}{|l|}{ Comorbidity } \\
\hline Hypertension & $86(29.55 \%)$ & $28(43.08 \%)$ & $58(25.66 \%)$ & 0.007 \\
\hline $\begin{array}{l}\text { Cardiovascular } \\
\text { diseases }\end{array}$ & $52(17.87 \%)$ & $20(30.77 \%)$ & $32(14.16 \%)$ & 0.002 \\
\hline Diabetes & $35(12.03 \%)$ & $9(13.85 \%)$ & $26(11.5 \%)$ & 0.609 \\
\hline Lung disease & $25(8.59 \%)$ & $10(15.38 \%)$ & $15(6.64 \%)$ & 0.027 \\
\hline Nephropathy & $13(4.47 \%)$ & $4(6.15 \%)$ & $9(3.98 \%)$ & 0.455 \\
\hline Hepatopathy & $18(6.19 \%)$ & $6(9.23 \%)$ & $12(5.31 \%)$ & 0.247 \\
\hline Cancer & $10(3.44 \%)$ & $3(4.62 \%)$ & $7(3.1 \%)$ & 0.554 \\
\hline $\begin{array}{l}\text { Immunodeficiency } \\
\text { diseases }\end{array}$ & $8(2.75 \%)$ & $3(4.62 \%)$ & $5(2.21 \%)$ & 0.296 \\
\hline \multicolumn{5}{|l|}{ Initial symptom } \\
\hline Fever & $235(80.76 \%)$ & $62(95.38 \%)$ & $173(76.55 \%)$ & 0.001 \\
\hline Dry cough & $131(45.02 \%)$ & $28(43.08 \%)$ & $103(45.58 \%)$ & 0.721 \\
\hline Fatigue & $126(43.3 \%)$ & $32(49.23 \%)$ & $94(41.59 \%)$ & 0.273 \\
\hline Muscle soreness & $75(25.77 \%)$ & $17(26.15 \%)$ & $58(25.66 \%)$ & 0.937 \\
\hline Stuffy nose & $12(4.12 \%)$ & $2(3.08 \%)$ & $10(4.42 \%)$ & 0.630 \\
\hline Runny nose & $14(4.81 \%)$ & $4(6.15 \%)$ & $10(4.42 \%)$ & 0.566 \\
\hline Chilling & $53(18.21 \%)$ & $12(18.46 \%)$ & $41(18.14 \%)$ & 0.953 \\
\hline Sore throat & $41(14.09 \%)$ & $6(9.23 \%)$ & $35(15.49 \%)$ & 0.201 \\
\hline Headache & $33(11.34 \%)$ & $13(20 \%)$ & $20(8.85 \%)$ & 0.012 \\
\hline Breath shortness & $65(22.34 \%)$ & $24(36.92 \%)$ & $41(18.14 \%)$ & 0.001 \\
\hline Chest tightness & $85(29.21 \%)$ & $30(46.15 \%)$ & $55(24.34 \%)$ & 0.001 \\
\hline \multirow[t]{2}{*}{ Anorexia } & $60(20.62 \%)$ & $18(27.69 \%)$ & $42(18.58 \%)$ & 0.110 \\
\hline & & Page $7 / 17$ & & \\
\hline
\end{tabular}




\begin{tabular}{lllll}
\hline Vomiting & $7(2.41 \%)$ & $1(1.54 \%)$ & $6(2.65 \%)$ & 0.605 \\
\hline Diarrhea & $47(16.15 \%)$ & $9(13.85 \%)$ & $38(16.81 \%)$ & 0.567 \\
\hline Chest CT findings & & & & \\
\hline $\begin{array}{l}\text { Unilateral lung } \\
\text { involvement }\end{array}$ & $27(9.28 \%)$ & $3(4.62 \%)$ & $24(10.62 \%)$ & 0.141 \\
\hline $\begin{array}{l}\text { Bilateral lung } \\
\text { involvement }\end{array}$ & $264(90.72 \%)$ & $62(95.38 \%)$ & $202(89.38 \%)$ & \\
\hline
\end{tabular}

Of the 291 patients, 65 (22.34\%) patients were included in the disease progression group when the disease progressed to a serious or critical state during the treatment; $226(77.66 \%)$ patients who did not progress were included in the improvement group. The median time from admission to disease progressed was 5.0 days (2.0-7.0), whereas the median time from illness onset (i.e., before admission) to disease progressed was 10.0 days (9.0-14.0).

Compared with improvement group, the patients of progression group were significantly older $(P=0.030)$ and male dominated $(P=0.003)$. Meanwhile, the patients of progression group have more underlying comorbidities $(P<0.001)$ including hypertension $(P=0.007)$, cardiovascular diseases $(P=0.002)$, and lung diseases $(P=0.027)$, higher incidence of fever $(P=0.001)$, headache $(P=0.012)$, breath shortness $(P=$ $0.001)$, and chest tightness $(P=0.001)$. There was no significant difference in duration from illness onset to first admission between the two groups $(P>0.05)$.

\section{Laboratory indices}

Compared with the improvement group, the patients in the progression group had decreased peripheral blood lymphocyte count $(P<0.001)$ (Table 2), aspartate aminotransferase $(P<0.001)$, serum creatinine $(P$ $=0.001)$, Urea nitrogen $(P<0.001)$, C-reactive protein $(P<0.001)$, interleukin-6(IL-6) $(P<0.001)$, creatine kinase $(P<0.001)$, lactate dehydrogenase $(P<0.001)$, Alpha hydroxybutyric dehydrogenase $(P<0.001)$, and $d$-dimer $(P<0.001)$ increased. Patients enrolled in progression group who were discharged showed the progress from progression to improvement on CT scan (Figure 1).

\section{Table 2: Laboratory findings on admission.}


per L

Lymphocyte count, $\times 10^{9}$ per $L$

$<1.1 \times 10^{9} / \mathrm{L}$

Alanine aminotransferase,

U/L

Aspartate aminotransferase
U/L

$>40 \mathrm{U} / \mathrm{L}$

Creatine, $\mu \mathrm{mol} / \mathrm{L}$

$>110 \mu \mathrm{mol} / \mathrm{L}$

Urea nitrogen,mmol/L

Uric acid, $\mu \mathrm{mol} / \mathrm{L}$

D-dimer, $\mathrm{ng} / \mathrm{ml}$

$>243 \mathrm{ng} / \mathrm{ml}$

Creatine kinase, $\mathrm{U} / \mathrm{L}$

$>300 \mathrm{U} / \mathrm{L}$

Creatine kinase isoenzyme,

$\mathrm{U} / \mathrm{L}$

Lactate dehydrogenase, U/L

$>225 \mathrm{U} / \mathrm{L}$

Alpha hydroxybutyric

dehydrogenase,IU/L

$>195 \mathrm{U} / \mathrm{L}$

Troponin,ng/ml

$>0.02 \mathrm{ng} / \mathrm{ml}$

Myoglobin,ng/ml
Total $(n=291)$

4.85(3.80-

6.10)

progression

group $(n=65)$

4.90(3.70-6.60)

$0.89(0.59-1.20)$

1.19(0.88-

1.59)

132(45.36\%)

21.5(15-35)

29(22-39)

65(22.34\%)

65(53-78)

10(3.44\%)

4.08(3.35-

5.16)

251(203-307) 254(214-326)

258(143.5-389)

140(81.5-

272)

$74(25.43 \%) \quad 30(46.15 \%)$

116(80-181)

$28(9.62 \%)$

17(15-20)

208(179-256)

268.5(213.5-

353.5)

$114(39.18 \%) \quad 44(67.69 \%)$

217(176.5-299)

168(143-214)

$81(27.84 \%)$

0.006(0.004-

$0.011)$

$33(50.77 \%)$

$0.009(0.006-$

$0.018)$

$23(7.90 \%)$

28.1(21-

50.93)

10(15.38\%) improvement

group $(n=226)$

$P$ value

4.80(3.90-6.00)

0.814

$1.25(0.95-1.65)$

$<0.001$

$86(38.05 \%)$

$<0.001$

21(15-34)

0.078

27(22-34)

$<0.001$

41(18.14\%)

0.001

63(51-76)

0.001

$3(1.33 \%)$

$<0.001$

3.87(3.25-4.92)

$<0.001$

250(201-304)

0.304

125(77-216)

$<0.001$

$44(19.47 \%)$

$<0.001$

$<0.001$

0.006

16(7.08\%)

0.091

17(14-20)

198(172-233)

$<0.001$

$<0.001$

$<0.001$

160(141-193)

48(21.24\%)

$<0.001$

0.006(0.004-0.009)

0.001

0.011

13(5.75\%)

$<0.001$ 


\begin{tabular}{lllll}
$>\mathbf{1 0 0} \mathbf{~ n g} / \mathrm{ml}$ & $30(10.31 \%)$ & $16(24.62 \%)$ & $14(6.19 \%)$ & $<0.001$ \\
\hline Interleukin-6, pg/ml & $9.6(3.3-27.5)$ & $29.8(12.7-53.1)$ & $6.95(2.8-20)$ & $<0.001$ \\
\hline$>\mathbf{4 0} \mathbf{~ p g / m l}$ & $34(11.68 \%)$ & $18(27.69 \%)$ & $16(7.08 \%)$ & $<0.001$ \\
\hline Procalcitonin,ng/ml & $\begin{array}{l}0.04(0.03- \\
0.07)\end{array}$ & $0.08(0.06-0.14)$ & $0.04(0.03-0.07)$ & $<0.001$ \\
\hline C-reactive protein,mg/L & $\begin{array}{l}10(4.87- \\
26.72)\end{array}$ & $28.2(9.3-55.06)$ & $10(4.015-16.095)$ & $<0.001$ \\
\hline$>30 \mathrm{mg} / \mathrm{L}$ & $60(20.62 \%)$ & $27(41.54 \%)$ & $33(14.60 \%)$ & $<0.001$ \\
\hline $\begin{array}{l}\text { Erythrocyte sedimentation } \\
\text { rate,mm/h }\end{array}$ & $20(11-40)$ & $23(11-55.5)$ & $20(10.5-37)$ & 0.159
\end{tabular}

\section{Treatments and outcomes}

Among the 291 patients, the most common treatment was antivirals including lopinavir/ritonavir, Interferon, Arbidol, and Ribavirin combined with Traditional Chinese Medicine (Table 3). The proportion of patients treated with lopinavir/ritonavir, glucocorticoid, and antibacterial were higher in the progression group than improvement group (all $\mathrm{P}<0.05$ ).

\section{Table 3: Treatments and Outcomes.}




\section{Total $(n=291) \quad$ progression

\section{Treatment}

\begin{tabular}{lllll}
\hline Lopinavir/ritonavir & $146(50.17 \%)$ & $40(61.54 \%)$ & $106(46.9 \%)$ & 0.038 \\
\hline Interferon & $175(60.14 \%)$ & $45(69.23 \%)$ & $130(57.52 \%)$ & 0.089 \\
\hline Arbidol & $54(18.56 \%)$ & $10(15.38 \%)$ & $44(19.47 \%)$ & 0.455 \\
\hline Ribavirin & $157(53.95 \%)$ & $32(49.23 \%)$ & $125(55.31 \%)$ & 0.386 \\
\hline $\begin{array}{l}\text { Traditional Chinese } \\
\text { medicine }\end{array}$ & $234(80.41 \%)$ & $54(83.08 \%)$ & $180(79.65 \%)$ & 0.539 \\
\hline Glucocorticoid & $129(44.33 \%)$ & $57(87.69 \%)$ & $72(31.86 \%)$ & $<0.001$ \\
\hline Antibacterial & $275(94.50 \%)$ & $65(100 \%)$ & $210(92.92 \%)$ & 0.027 \\
\hline Thymosin & $209(71.82 \%)$ & $48(73.85 \%)$ & $161(71.24 \%)$ & 0.681 \\
\hline Respiratory support & & & & $<0.001$ \\
\hline Nasal cannula & $260(89.35 \%)$ & $47(72.31 \%)$ & $213(94.25 \%)$ & $<0.001$ \\
\hline High-flow nasal cannula & $2(0.69 \%)$ & $2(3.08 \%)$ & $0(0.00 \%)$ & $<0.001$ \\
\hline Noninvasive ventilator & $13(4.47 \%)$ & $13(20.00 \%)$ & $0(0.01 \%)$ & \\
\hline Invasive mechanical & $2(0.69 \%)$ & $2(3.08 \%)$ & $0(0.02 \%)$ & \\
\hline ventilation & & & $18(13,23)$ & \\
\hline ECMO & $1(0.34 \%)$ & $1(1.54 \%)$ & $0(0.03 \%)$ & \\
\hline Prognosis & & & $226(100.00 \%)$ & \\
\hline Discharge & $279(95.89 \%)$ & $53(81.54 \%)$ & $0.00 \%)$ & \\
\hline Death & $12(14.11 \%)$ & $12(18.46 \%)$ & & \\
\hline Hospitalization day & $28(19,35)$ & & \\
\hline
\end{tabular}

In addition, respiratory support was used for preventing acute hypoxic respiratory failure. 13 patients used noninvasive ventilator, and two patients used invasive ventilator. Moreover, one patient was treated with extracorporeal membrane oxygenation (ECMO). The progression group had more severe hypoxia and was significantly more likely to receive higher levels of respiratory support compared to the improvement group $(\mathrm{P}<0.001)$. 
By Mar 16, 2020, 279 (95.89\%) patients had been discharged and 12 (4.11\%) patients had died. Hospitalization day were longer in the progression group than that in the improvement group $(P<0.001)$.

\section{Risk factors for in-hospital progression in COVID-19 patients}

The factors with statistical difference between the two groups included age, gender, history of cardiovascular, lymphocyte count, IL6, d-dimer, lactate dehydrogenase on admission was included in the multivariate logistic regression analysis. The results indicated that male (odds ratio [OR], 2.333; $\mathrm{P}=$ $0.020)$, preexisting cardiovascular disease $(O R, 2.433 ; P=0.039)$, lymphopenia $(O R, 3.482 ; P<0.001)$, and elevated levels of blood IL-6 (OR, 2.669; P =0.033), D-dimer (OR, 2.829; P =0.003), lactate dehydrogenase $(\mathrm{OR}, 2.855 ; \mathrm{P}=0.002)$ were associated with in-hospital progression(Table 4).

Table 4: Risk factors associated progression of COVID-19 patients.

$P$ value Multivariable OR $\quad 95 \% \mathrm{Cl}$

Age (years)

0.705

0.996

0.973-1.019

Sex (male vs. female)

$0.020 \quad 2.333$

$1.135-4.395$

Cardiovascular diseases (yes vs. no)

0.039

2.433

$1.044-5.671$

Lymphocyte count $\left(<1.1 \times 10^{9} /\right.$ L vs. $\geq 1.1 \times 10^{9} / \mathrm{LV}$

$<0.001$

3.482

$1.783-6.799$

IL-6 (>40 pg/ml vs. $\leq 40 \mathrm{pg} / \mathrm{ml}$

$0.033 \quad 2.669$

$1.084-6.574$

D-dimer (>243 ng/ml vs. $\leq 243 \mathrm{ng} / \mathrm{ml} \rrbracket$

$0.003 \quad 2.829$

$1.420-5.636$

lactate dehydrogenase (>225U/L vs. $\leq 225 \mathrm{U} / \mathrm{L}$

$0.002 \quad 2.855$

$1.458-5.591$

\section{Discussion}

We described a cohort of 291 adult patients who were ordinary COVID-19 cases when admitted and all patients had outcomes that 279 were discharged (95.89\%), 12 died (4.11\%), 65 (22.3\%) had been recorded COVID-19 progressing at least once, and 226 (77.7\%) had been recorded COVID-19 improving all the time during hospitalization. The time from onset to first admission was 7.0 days, 19 days of hospitalization. Common symptoms at onset of illness were fever, dry cough, and fatigue, while higher proportion of progression cases showed fever, headache, shortness of breath, chest tightness. In this study, we were able to identify some clinical and laboratory characteristics on presentation that were associated with the COVID-19 progression. Considering the total number of cases in progression group and to avoid over fitting in the model, eight variables were chosen for multivariate logistic analysis based on previous reports and clinical constraints. Previous studies have reported that older age and elevated ddimer on admission can increased the risk of COVID-19 death ${ }^{12}$. Additionally, males, lymphopenia, cardiovascular disease, and elevated levels of blood IL6, lactate dehydrogenase have been more commonly observed in sever or critical or non-surviving patients with COVID-196,7,12,13 .Therefore, we 
chose age, male, cardiovascular disease, lymphocyte count, d-dimer, IL6, lactate dehydrogenase as the seven variables for our multivariable logistic regression model. Finally, the results showed males, preexisting cardiovascular disease, lymphopenia, and elevated levels of blood IL6, lactate dehydrogenase, and d-dimer were independent predictors in the multivariable analysis.

Yang et al reported findings from 52 sever cases suggested that men and people of an older age are more likely to progressive to death than women or those of a younger age ${ }^{14}$. And Fan et al reported similar findings from 101 death of COVID-19 ${ }^{13}$. Previous studies have also reported that advanced age, male sex were associated with a high case-fatality rate and independent predictors of adverse outcome in SARS ${ }^{15-}$ 17. The results in our study partially supported previous reports that advanced age and male sex were associated with COVID-19 progression in univariable analysis, male sex was also independent predictor in multivariable analysis, but advanced age was not. The age-dependent defects in physical condition and immune system were more likely to lead to poor outcomes of viral pneumonia ${ }^{18,19}$. The probable reason of our result maybe because only ordinary cases on admission enrolled in our study, of which 49 $(16.8 \%)$ were older than 70 years. Older patients classified as severe or critical cases when admitted were excluded from our study, while they more easily progressed to adverse outcomes during hospitalization ${ }^{20}$.

Our results found that patients with preexisting cardiovascular disease more easily developed into severe condition and showed more severe damage to heart which was evaluated by significantly elevated myoglobin and creatine kinase. Recently a meta-analysis including six studies with 1527 COVID-19 patients reported that the incidence of cardia-cerebrovascular diseases was three-folds higher in ICU/sever cases than in non-ICU/ sever counterparts, and COVID-19 can, in turn, aggravate the damage to the heart ${ }^{21}$. This result just proved our findings. Full-genome sequencing and phylogenic analysis indicated SARS-CoV-2 has a similar receptor-binding domain structure to that of SARS-CoV, which binds to the angiontensin-coverting enzyme 2 (ACE2) in humans ${ }^{1}$. ACE2 expressed on myocyte and vascular endothelial cells ${ }^{22,23}$, and some studies found circulating ACE2 has been shown to be increased in patients with cardiovascular disease and activity increased in patients with left ventricular systolic dysfunction ${ }^{24,25}$. So there are theoretical potential possibilities of direct cardiac involvement and severe infection by the virus.

High initial lactate dehydrogenase, IL6, d-dimer, and lymphopenia were independent predictors for progression in multivariable analysis. Since high lactate dehydrogenase levels are often seen in association with tissue damage. This finding indicates more extensive tissue injury may have happened in progression group when admitted. Early studies have shown that increased levels of circulating cytokines in serum, such as IL6, IL-12, MCP1, were associated with pulmonary inflammation and extensive lung damage in SARS patients ${ }^{26}$. Zhou et al reported findings from 191 COVID-19 cases showed that elevated IL6 and lactate dehydrogenase in non-survivors happened during the whole hospitalization, but they didn't evaluate the role of IL6 and lactate dehydrogenase playing in predicting inhospital death. In our study, we found high initial lactate dehydrogenase, and IL 6 are independent risk factors to predict in-hospital COVID-19 progression. This found should be attached importance to and 
need further evaluated by more studies. It was common to be found increased coagulation activity in patients with pneumonia, marked by increased level of d-dimer. D-dimer greater than $1 \mathrm{mg} / \mathrm{L}$ was associated with fatal outcome of COVID-19 ${ }^{12}$. In this study, we found high initial d-dimer is associated with COVID-19 progression. Lymphopenia has been reported in SARS, Ebola, and COVID-19, thus there seems to be a connection between viral infections and lymphopenia, whether the viruses directly causes lymphopenia is still a question ${ }^{27}$. Many studies reported that lymphopenia was a high-risk factor for adverse outcomes of SARS ${ }^{15,28}$, and our result shows lymphopenia is an independent predictor for COVID-19 progression.

In our study, all patients received antiviral treatment, but the types of drugs used varied. Given the retrospective nature of our study, it is difficult to determine whether there is any therapeutic benefit to the treatment regimens used in treating COVID-19, specifically lopinavir/ritonavir, glucocorticoids. Recently, the result of the randomized clinical trial for lopinavir/ritonavir (ChiCTR2000029308) has been published that no benefit was observed among in-hospital patients with severe COVID-1929. Therefore, the effectiveness and safety of lopinavir/ritonavir needs to be evaluated by more research. There are some disagreements between using and not using glucocorticoids during the treatment of viral pneumonia, due to the conflict of anti-inflammatory and immunosuppression ${ }^{30,31}$. So, the time and dose of glucocorticoids use is important for disease development.

Our study still has some limitations. Firstly, certain data may have based on patient memory, such as timing of onset of symptoms that may be affected by recall bias, and certain laboratory tests were not done in all patients, such as troponin and myoglobin, that may be underestimated their role in predicting disease progression. Secondly, lack of effective antivirals, and high-dose glucocorticoids use might have contributed to the progression in some patients.

\section{Conclusions}

As we described that most COVID-19 patients were just ordinary cases when admitted, nearly $23 \%$ of those had been recorded progressing or even death. Early detection and treatment is important for prevention of COVID-19 progression to severity and reduction of mortality. In this study, male, preexisting cardiovascular disease, lymphopenia, elevated IL-6, and lactate dehydrogenase, d-dimer on admission were potential risk factors associated with progression which could help clinicians to identify in-hospital progression at early stage to optimize medical treatment.

\section{Declarations}

Funding: None.

Author contributions: $A Y, Y Z$, and $X F$ had the idea for and designed the study and had full access to all of the data in the study. AY took the responsibility for the integrity of the data and the accuracy of the data analysis. YZ and FX drafted the paper. Yan Z, FZ, LZ, JL, CZ, LT, ZL and YX collected the data. HL, Yong Z, 
and YT checked all data and all authors critically revised the manuscript and gave final approval for the version to be published.

Competing interests: The authors declare no competing interests.

Acknowledgement: We thank the nurses and physicians who provided care for the patients at General Hospital of Central Theatre Command.

\section{References}

1 Lu, R. et al. Genomic characterisation and epidemiology of 2019 novel coronavirus: implications for virus origins and receptor binding. Lancet 395, 565-574, doi:10.1016/S0140-6736(20)30251-8 (2020).

2 Phan, L. T. et al. Importation and Human-to-Human Transmission of a Novel Coronavirus in Vietnam. The New England journal of medicine 382, 872-874, doi:10.1056/NEJMc2001272 (2020).

3 Chan, J. F. et al. A familial cluster of pneumonia associated with the 2019 novel coronavirus indicating person-to-person transmission: a study of a family cluster. Lancet 395, 514-523, doi:10.1016/S01406736(20)30154-9 (2020).

4 Novel Coronavirus (COVID-19) Situation., <https://experience.arcgis.com/experience/685d0ace521648f8a5beeeee1b9125cd> (

5 National Health Commission of the People's Republic of China. Chinese management guideline for COVID-19 (version 7.0). <http://www.gov.cn/zhengce/zhengceku/2020-03/04/content_5486705.htm>(

6 Huang, C. et al. Clinical features of patients infected with 2019 novel coronavirus in Wuhan, China. Lancet 395, 497-506, doi:10.1016/S0140-6736(20)30183-5 (2020).

7 Wang, D. et al. Clinical Characteristics of 138 Hospitalized Patients With 2019 Novel CoronavirusInfected Pneumonia in Wuhan, China. Jama, doi:10.1001/jama.2020.1585 (2020).

8 Chen, N. et al. Epidemiological and clinical characteristics of 99 cases of 2019 novel coronavirus pneumonia in Wuhan, China: a descriptive study. Lancet 395, 507-513, doi:10.1016/S01406736(20)30211-7 (2020).

9 Liu, W. et al. Analysis of factors associated with disease outcomes in hospitalized patients with 2019 novel coronavirus disease. Chinese medical journal, doi:10.1097/CM9.0000000000000775 (2020).

10 Tian, S. et al. Characteristics of COVID-19 infection in Beijing. The Journal of infection, doi:10.1016/j.jinf.2020.02.018 (2020).

11 Chen, J. et al. Clinical progression of patients with COVID-19 in Shanghai, China. The Journal of infection, doi:10.1016/j.jinf.2020.03.004 (2020). 
12 Zhou, F. et al. Clinical course and risk factors for mortality of adult inpatients with COVID-19 in Wuhan, China: a retrospective cohort study. Lancet, doi:10.1016/S0140-6736(20)30566-3 (2020).

13 Fan., H. et al. Retrospective Analysis of Clinical Features in 101 Death Cases with COVID-19. medRxiv preprint, doi: https://doi.org/10.1101/2020.03.09.20033068.

14 Yang, X. et al. Clinical course and outcomes of critically ill patients with SARS-CoV-2 pneumonia in Wuhan, China: a single-centered, retrospective, observational study. The Lancet. Respiratory medicine, doi:10.1016/S2213-2600(20)30079-5 (2020).

15 Leung, G. M. et al. The epidemiology of severe acute respiratory syndrome in the 2003 Hong Kong epidemic: an analysis of all 1755 patients. Annals of internal medicine 141, 662-673, doi:10.7326/00034819-141-9-200411020-00006 (2004).

16 Lee, N. et al. A major outbreak of severe acute respiratory syndrome in Hong Kong. The New England journal of medicine 348, 1986-1994, doi:10.1056/NEJMoa030685 (2003).

17 Booth, C. M. et al. Clinical features and short-term outcomes of 144 patients with SARS in the greater Toronto area. Jama 289, 2801-2809, doi:10.1001/jama.289.21.JOC30885 (2003).

18 Cilloniz, C. et al. Impact of age and comorbidity on cause and outcome in community-acquired pneumonia. Chest 144, 999-1007, doi:10.1378/chest.13-0062 (2013).

19 Opal, S. M., Girard, T. D. \& Ely, E. W. The immunopathogenesis of sepsis in elderly patients. Clinical infectious diseases : an official publication of the Infectious Diseases Society of America $\mathbf{4 1}$ Suppl 7, S504-512, doi:10.1086/432007 (2005).

20 Li, Y., Song, J., Xie, Z., Liu, M. \& Sun, K. Long noncoding RNA colorectal neoplasia differentially expressed alleviates sepsis-induced liver injury via regulating miR-126-5p. IUBMB life 72, 440-451, doi:10.1002/iub.2230 (2020).

$21 \mathrm{Li}, \mathrm{B}$. et al. Prevalence and impact of cardiovascular metabolic diseases on COVID-19 in China. Clinical research in cardiology : official journal of the German Cardiac Society, doi:10.1007/s00392-020-01626-9 (2020).

22 Gallagher, P. E., Ferrario, C. M. \& Tallant, E. A. Regulation of ACE2 in cardiac myocytes and fibroblasts. American journal of physiology. Heart and circulatory physiology 295, H2373-2379, doi:10.1152/ajpheart.00426.2008 (2008).

23 Mendoza-Torres, E. et al. ACE2 and vasoactive peptides: novel players in cardiovascular/renal remodeling and hypertension. Therapeutic advances in cardiovascular disease 9, 217-237, doi:10.1177/1753944715597623 (2015). 
24 Anguiano, L., Riera, M., Pascual, J. \& Soler, M. J. Circulating ACE2 in Cardiovascular and Kidney Diseases. Current medicinal chemistry 24, 3231-3241, doi:10.2174/0929867324666170414162841 (2017).

25 Epelman, S. et al. Soluble angiotensin-converting enzyme 2 in human heart failure: relation with myocardial function and clinical outcomes. Journal of cardiac failure 15, 565-571, doi:10.1016/j.cardfail.2009.01.014 (2009).

26 Wong, C. K. et al. Plasma inflammatory cytokines and chemokines in severe acute respiratory syndrome. Clinical and experimental immunology 136, 95-103, doi:10.1111/j.1365-2249.2004.02415.x (2004).

27 Panesar, N. S. What caused lymphopenia in SARS and how reliable is the lymphokine status in glucocorticoid-treated patients? Medical hypotheses 71, 298-301, doi:10.1016/j.mehy.2008.03.019 (2008).

28 Tai, D. Y. et al. Clinical features and predictors for mortality in a designated national SARS ICU in Singapore. Annals of the Academy of Medicine, Singapore 32, S34-36 (2003).

29 Cao, B. et al. A Trial of Lopinavir-Ritonavir in Adults Hospitalized with Severe Covid-19. The New England journal of medicine, doi:10.1056/NEJMoa2001282 (2020).

30 Torres, A. et al. Effect of corticosteroids on treatment failure among hospitalized patients with severe community-acquired pneumonia and high inflammatory response: a randomized clinical trial. Jama 313, 677-686, doi:10.1001/jama.2015.88 (2015).

$31 \mathrm{Kil}, \mathrm{H}$. R. et al. Early corticosteroid treatment for severe pneumonia caused by 2009 H1N1 influenza virus. Critical care 15, 413, doi:10.1186/cc10082 (2011).

\section{Figures}

\section{Figure 1}

Title: Chest computed tomography images of a 38-year-old patient with COVID-19 included in progression group. Legend: (A) Chest computed tomography images on admission day; (B) Chest computed tomography images on day 11 after admission; (C) Chest computed tomography images 17 day after admission; (D) Chest computed tomography images 18 day after discharge. 